\title{
Improving V2X-6G network capacity using a new UAV-based approach in a Cloud/ICN architecture, case Study: VANET network
}

\author{
Abdeslam Houari $^{1 *}$, and Tomader Mazri ${ }^{1}$ \\ ${ }^{1}$ Laboratory of Electrical and Telecommunication Engineering, Ibn Tofail science University, Kenitra, Morocco
}

\begin{abstract}
G}$ of mobile networks plays a crucial role in improving the capacity and enhancing the quality of services of Vehicle-to-Everything (V2X) based networks evolving in an intelligent environment. VANET is a promising project in the intelligent transportation field using V2X communications. The emergence of several $5 \mathrm{G}$ and $6 \mathrm{G}$ technologies has raised several challenges for scientists and researchers to allow vehicles and road users to enjoy several services while ensuring their safety on the road. Among these technologies, the unmanned aerial vehicle (UAV), which can perform different tasks for road users and vehicle drivers such as data caching, packet relaying and processing. In this article, we present a new approach based on 6G Unmanned Aerial Vehicles (UAV) technology on a vehicular cloud architecture while exploiting the exchange support of information-centric networking (ICN) for the improvement of network capacity.
\end{abstract}

\section{Introduction}

V2X communication has attracted the attention of the scientific community and industry, especially those operating in the automotive field. As a communication medium for Intelligent Transport Systems (ITS), V2X includes a large number of wireless communication technologies, namely vehicle-to-vehicle, vehicle-toinfrastructure, and vehicle-to-pedestrian. Other types of communications have been subsequently introduced that are less known than those previously mentioned, such as VRU for communications with vulnerable road users as well as communications with the cloud network V2N[1]. The purpose of the scientists is to make V2X supported by $6 \mathrm{G}$ Mobile networks and wireless systems a key element for the connection of autonomous vehicles in smart cities. Besides, the integration of V2X will improve the road users' safety and make it more comfortable, decrease considerably the air pollution due to the emission of $\mathrm{CO} 2$ by vehicles on the road, and allow the deployment of several advanced applications in the field of road transportation. The implementation of these applications is enabled by using V2X communication technologies, such as cellular V2X and dedicated shortrange (DSRC). This last one is a wireless communication technology devoted to the automotive sector and applications related to intelligent transportation systems, considered for a long time as the unique technology for V2X communications, used in high mobility and dense network conditions.

VANET project is an important project related to intelligent transport systems and more precisely to systems based on V2X communications. VANET is a form of MANET network where the nodes are vehicles (intelligent), the purpose of this system is to allow vehicles to communicate with each other in order to identify different issues that may occur on the road. Vehicles can communicate with each other via the V2V mode using devices such as OBUs, and with the road infrastructure via the V2I mode in order to benefit from multiple services such as internet access. The high node mobility is the major feature of the VANET, due to the vehicles' high speed and their random and unpredictable movements which directly impact the communication time and the network topology which becomes extremely dynamic.

On the other hand, cloud computing is now widely used by many companies and individuals for many purposes, from the simple transfer of data and services to remote servers to the exploitation of entire platforms. Indeed, several companies have started to set up their own cloud service infrastructure which has attracted many users desiring to explore this new type of service.

The automotive sector also relies on Cloud technology to improve its existing services and to diversify the available functionalities. Given the considerable processing power and data storage capacity of vehicles, automotive researchers intend to include them in a vehicle-specific cloud approach. In order to make them service producers, leading to the realization of a cloud service platform in which vehicles share their resources for the benefit of network vehicles or other users such as pedestrians or roadside authorities.

In the first part of this article, we introduce $\mathrm{V} 2 \mathrm{X}$ networks based on $6 \mathrm{G}$, in order to discover the benefits of the $6 \mathrm{G}$ in comparison with the $5 \mathrm{G}$ as well as the main technologies on which it is based in a V2X context. In the second part, we explore VANET networks with their different modes of communication and the challenges

* Corresponding author: abdeslam.houari@uit.ac.ma 
they face. In the two penultimate sections, we introduce the two Vehicular Cloud (VC) and Information-Centric Networking (ICN) architectures which will be employed in the proposed approach presented in the last section of this article. And we conclude with the conclusion.

\section{6G-V2X network}

\subsection{The reason for using $6 \mathrm{G}$ for V2X-based networks}

Despite the fact that $5 \mathrm{G}-\mathrm{V} 2 \mathrm{X}$ provides a better performance with different advanced features, however, this achievement is possible only through the massive exploitation of spectral and physical resources, while adopting the mechanism and architecture of LTE-based V2X systems[2]. Furthermore, it is expected that the number of autonomous vehicles will increase considerably in the near future due to urbanization, evolving life standards, and advanced technologies in different sectors. Consequently, several communication devices and applications will be released to facilitate the deployment of autonomous vehicles. All these evolutions will challenge V2X-based communications networks and largely impact their capacity, and new challenges will be raised for the scientific community to adapt latency, coverage, intelligence, and data rate to meet the new evolving requirements. In such a context, 5G-based V2X systems are not adapted to satisfy these different scenarios and requirements, since according to the studies that have been carried out in ITS projects, V2X communication systems can only provide a restricted intelligence. The desired transformation will be possible only from the newly released $6 \mathrm{G}$ wireless communication networks, which aims to use combinations of terrestrial and non-terrestrial wireless communication networks such as unmanned-aerialvehicle (UAV) and satellite. This will enhance the intelligence of V2X systems as well as the reliability of exchanges and the security of packet delivery, with very high data rate performance, and very fast wireless access, in addition to a wider, intelligent, and ecological 3D communication coverage[3]. Due to the heterogeneous composition of the network as well as the different communication use cases and the high quality of service requirements, new technologies are required to ensure intelligent decision-making in V2X systems.

\subsection{Overview of the $6 \mathrm{G}$}

With the advent of $5 \mathrm{G}$ mobile networks and the maturity of the technologies used, the attention of researchers has turned to the development of the next generation of mobile networks. Indeed, several countries have revealed their research programs concerning the development of the 6G. For example, the European Union at the end of 2017, has initiated a research project that is spread over 3 years that will focus on $6 \mathrm{G}$ technologies. The purpose is to study the next generation, focusing on channel modulation techniques, channel enhanced coding, and error detection. China has started (in the same year) the exploration of the $6 \mathrm{G}$ wireless communication systems in order to respond to the growing need of the internet of things (IoT), such as the development of sensing, image processing maps, or medical imaging. Recently the British government has invested significant amounts in the main techniques of the $6 \mathrm{G}$, such as the quantum technology studies for which they have invested an amount of 15 million euros (https://www.standard.co.uk/tech/quantum-

technologies), in other countries such as the United States, some universities have started researches about $6 \mathrm{G}$ wireless network based on terahertz, South Korea has initiated a project of the $6 \mathrm{G}$ on the network techniques non-terrestrial. For many researchers, the commercialization and market entry of $6 \mathrm{G}$ is only expected in about 10 years, based on the commercialization dates of the previous generations of wireless communication systems.

This new generation of mobile networks is expected to provide higher capacity than the previous $5 \mathrm{G}$, much lower latency to support the various applications that may emerge, such as intelligent natural disaster prediction systems, and systems of virtual reality (VR). $6 \mathrm{G}$ will fully exploit the technical architecture of $5 \mathrm{G}$, inheriting the various advances and potential of the $5 \mathrm{G}$ technologies, an optimized and decentralized network architecture that will change the way we see and design things.

There is a set of technologies that will enable the achievement of the 6G-V2X project as an autonomous, robust, intelligent service platform for ITS systems. The following section describes the most relevant technologies used, which will revolutionize the intelligent transportation industry, as well as the efficient management of devices in a connected environment.

\subsection{TECHNOLOGIES FOR 6G-V2X System}

Several $6 \mathrm{G}$ technologies are potentially relevant in the context of V2X systems (Fig. 1). Below is a list of the most popular ones.

\subsubsection{V2X supported by QC}

In a 6G-V2X network, it is expected that there would be a significant number of connected vehicles in a relatively dense environment, a more diversified range of applications, complex signal analysis, more need for reliability, computing power, low latency compared to $5 \mathrm{G}$ based V2X systems. That's why $6 \mathrm{G}-\mathrm{V} 2 \mathrm{X}$ networks need more processing resources that are normally available on the network. These resources will perform many complex tasks at an accelerated frequency. The emergence of quantum computing (QC) for wireless communication will support the processing and execution of complex algorithms in a reduced timeframe in order to improve V2X related services, such as the efficient processing of machine learning algorithms that require huge volumes of data and massive training. In similar use cases, traditional processing units are unable to satisfy this type of request, while the QC can accomplish it at a relatively low processing complexity while optimizing the consumed resources[4]. 


\subsubsection{BVC interfacing}

This new futuristic brain-controlled vehicle technology will allow the vehicle to be controlled by the human brain instead of the human's physical interaction with the vehicle, which will be very beneficial for humans with reduced mobility to drive a vehicle. this is possible by transforming brain activity signals into movement commands for the vehicle using a computerbrain interface. the aim also of the BVC is to include the human in the equation of autonomous driving of vehicles, by limiting the different cases of the uncertainty of decision making mainly in rural areas (not covered) or non-structured zones. Recent researches have demonstrated the validity of the BCV approach. For example, the authors[5] have developed a brain-based vehicle system, enabling the selection of vehicle destinations.

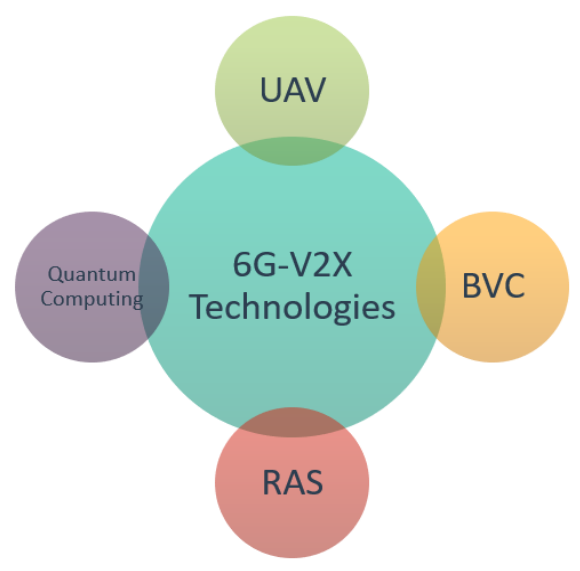

Fig. $1: 6 \mathrm{G}-\mathrm{V} 2 \mathrm{X}$ Technologies

For 6G-V2X networks, resource management radios (RRMs) play an important function in supplying V2X applications that respect the standards and satisfy the QoS requirements. Usually, it is the base stations that are in charge of the RRM in V2X cellular networks. However, there are several restrictions on the application and maintenance of RRM for the case of $6 \mathrm{G}-\mathrm{V} 2 \mathrm{X}$ networks. V2X networks in practice largely require multi-radio services in order to meet the QoS requirement. Resources from different technologies are involved in the decision-making of the RRM (such as the high data rate for mmWave). Besides, studies on RRM in a $5 \mathrm{G}$ context have focused on infrastructure-oriented communications. Furthermore, the problem will increase with the application of $6 \mathrm{G}$ in a V2X network due to the massive spread of mmWave as well as the resulting conflict between multiple V2X modes in addition to V2V communications and autonomous vehicle resource controls, in addition to the dynamic nature of the V2X network. To meet the various challenges of supporting multi-radio technologies and the complexity of processing and algorithms. Advanced resource allocation schemes are needed, which can be implemented with the support of a cross-layer model. For example [6] shows the importance of using intelligent multi-radio solutions to reliably provide good quality video in a highly mobile context.

\subsubsection{V2X assisted by UAV}

The ability to cover a wider area is one of the major features of the unmanned aerial vehicle (UAV), which can serve as a radio access point at the aerial level for the benefit of the V2X network based on 6G. UAVs can perform different tasks for road users and vehicle drivers such as data caching, packet relaying, and processing[7]. UAVs can also coordinate with static elements of the network infrastructure (road-side-unit, base station) to manage wireless network communications and improve the user experience. As a 3D mobile airborne motion unit, UAV can provide a variety of V2X applications, such as a) guiding rescue units to the accident location occurring on the road, b) supervising the traffic violations to assist the road authorities and finally c) issuing warning messages along the road not equipped with RSU[8]. Despite the advances undertaken in UAV technologies, there are several challenges in the field of UAV implementation for V2X systems. For instance, the fast-wireless communication between UAVs and vehicles on the ground is very challenging to maintain and to make reliable, due to the high mobility of UAVs and vehicles that make the communication channel very dynamic and unpredictable. In addition to the security and regularization problems that are still a subject of divergence in several countries. Without missing the lack of integration with similar communication networks as well as the battery consumption that limits its long-term employability.

In the following section, we focus on the VANET project which is a specific form of MANET network that uses V2X communications between its different nodes.

\section{VANET network}

In a smart city, a vehicle (or also called autonomous Vehicle), is designed to drive partially or entirely without human intervention. there are currently five levels of autonomy, the first level, for which the driver delegates some tasks while monitoring the vehicle, the fifth level, the vehicle is assumed to be $100 \%$ autonomous. The autonomous vehicle depends mainly on the sensors embedded in it, in addition to the processing and detection algorithms and powerful machine learning systems. The role of the sensors is to discover their surroundings. the cameras and LiDar detect all the events that occur around them, and are considered as the vehicle's eyes, they inform the vehicle if there is an obstacle, a change of traffic light, or any other incident occurring on the road; all the data collected will be processed internally by algorithms that convert the incoming information into action, such as avoiding an obstacle or making a stop in a traffic light or changing direction in the case of an accident.

Researchers in the automotive field, have proposed several sensors and intelligent devices to be integrated on board the vehicle so that it can be autonomous and drive safely by interpreting the incoming information in order to prevent any potential obstacle that could block the progress of the vehicle. In order for a vehicle to be aware of its surroundings, it must have on average eight cameras positioned all around the vehicle, in addition to a LiDar (or RADAR) system for the detection of 
eventual obstacles, and finally a GPS for locating the vehicle on the road network and facilitating the search for a given position.

\subsection{Overview of VANET}

VANET is a promising project in the field of intelligent transportation. It represents a specific case of MANET networks enabling inter-node communication as well as internal management with no dependency on any external infrastructure. The aim is to connect vehicles in order to share data and resources to better discover their surroundings and to deal with any unexpected events that may arise on the road, such as breakdowns, traffic jams, or road accidents, which are recurrent problems for road users. The VANET network topology is constantly changing due to the high mobility of vehicles moving in and out of the clusters in a very short period. As a direct consequence of this behavior, the connection links become unstable and can be broken at any time, causing in some cases the loss of the exchanged data frames. the other distinctive feature of this network is its unlimited size since there is no geographical limitation, unlike other MANET networks which are geographically limited and suffer from energy and resource problems, which is not the case for VANET.

In order to communicate with each other, the vehicles are equipped with On-Board Unit (OBU), this device is placed onboard the vehicle and combined with the RSU (Road-side-unit), it allows the vehicle to collect information from the sensors installed onboard and to share them with other vehicles in the network, the connection between the OBUs and the RSU is carried out through an IEEE 802.11p wireless link[9].

Regarding the RSU, it is a wireless communication device placed alongside the road, allowing the extension of the communication range, and also serves as a communication and connection support for the vehicles passing under its coverage.

\subsection{Communication modes in VANET}

In VANET network, the vehicles communicate with each other via the V2V (vehicle-to-vehicle) mode (Fig. 2), the architecture dedicated to this type of communication is decentralized, this mode is efficient especially for sending alert messages and sharing data on road conditions, but this mode is subject to several dysfunctions due to the high mobility of the vehicles which causes in many cases the interruption of connections and consequently the data loss.

The data exchange between the vehicles on the one hand and the infrastructure (RSU, base station, satellite) on the other hand are performed through the V2I mode (vehicle-to-infrastructure). This mode ensures an optimized use of the common resources besides the connection status which is more stable compared with the V2V mode[10].

The third mode of communication, which not only links vehicles and infrastructure but also includes cellular networks and other devices and equipment, is known as the Vehicle-to-everything (V2X) mode, which is composed of V2V, V2I in addition to the V2P Vehicleto-Pedestrian and Vehicle-to-Device (V2D) modes. In this kind of ecosystem, vehicles interact with a variety of stakeholders, pedestrians via smartphones, and data centers and servers via cellular networks (base station).

\subsection{VANET Challenges}

VANET faces several challenges in setting up a reliable and secure exchanging system, the most critical ones are security and privacy, mainly because data packets are exchanged between network nodes and anyone can Intercept them and use their contents for malicious purposes that potentially endanger the life of road users. Security should satisfy particular requirements for the normal functioning of any system as well as the continuity of the provided services to its clients. Failure to satisfy any of these requirements may cause fatal consequences such as data loss or system crashes.

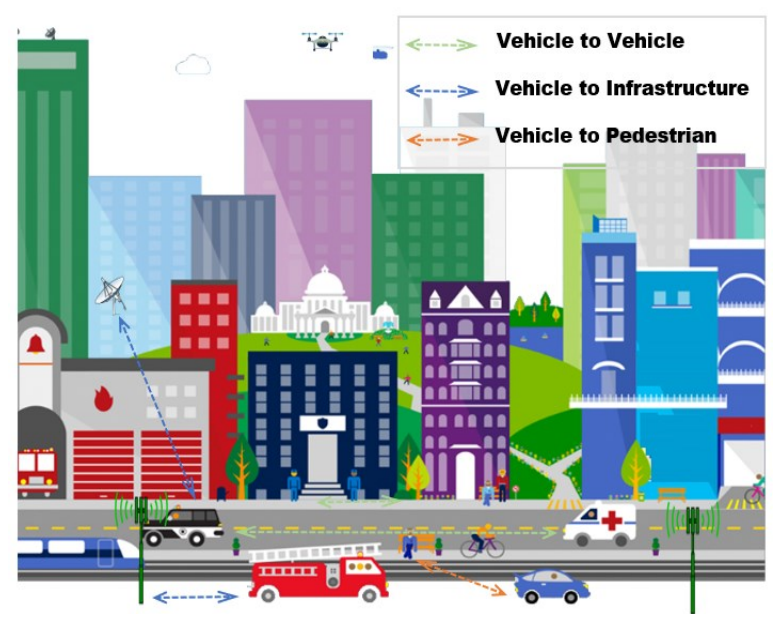

Fig. 3 : Communication modes in VANET

Other prerequisites are specific to VANET networks (compared to other MANET networks) such as data integrity, authentication and confidentiality. Once a vehicle is authenticated, it is assigned a unique identifier, preventing it from appearing as more than one vehicle, and consequently usurping the identities of other vehicles[11]. Data integrity ensures that the data packets have not been corrupted or modified during their transmission to the destination node. Regarding confidentiality, the aim is to ensure that the communication links are reliable, the best-known method used for this purpose is the encryption of the messages, this guarantees that only the recipient of the message is eligible to consult its content. Despite these measures, communicating in an open space, lead to the intrusion of malicious nodes that can corrupt the transmitted data. For several researchers specialized in this field, the major challenge is to prevent attackers from accessing the information transmitted by vehicles in order to maintain the reliability of communication within

the network and avoid the loss of sensitive data related to road conditions and the personal data of passengers and 
drivers, for this purpose, several methods have been proposed, the best known is the RGTE (ReputationBased Global Trust Establishment)[12], this method is based on statistical laws approach in order to share only trustful information, it also permits the detection of malicious nodes via an innovative mechanism, unfortunately, this method is not applicable in the case of high node mobility.

\section{Cloud Computing in VANET Network}

Cloud computing is widely regarded as service computing, based on the concept of paying for what you consume and use (pay-as-you-go), which in reality reflects the scenario of our daily life, in which we use a set of services such as electricity and water, internet and at the end of each month we pay for what we have consumed.

As a result, $\mathrm{CC}$ has emerged as a concept providing fast on-demand access to a set of shared resources organized in three main layers[13], the top layer is called SaaS (Software as a Service), the role of this layer is to deliver applications to customers, both individuals and enterprises, the provider is fully in charge of the maintenance and availability of the software.

The second type of service is the PaaS (Platform as a Service), in which instead of installing software and development or other tools on the host machines, all the required technical environment (including tools and software) is delivered as a service. this enables customers to work remotely on their tasks using only the services provided, while the provider is responsible for the availability of the network of servers as well as the storage space allocated for each service, the known example of this type of solution is Google App Engine.

Finally, at the bottom of the stack of layers, there is the IaaS (Infrastructure as a Service)[14]. In this case, physical resources are offered as a service, including servers and the development tools required to build a technical environment from scratch. clients have at their disposal important resources according to the allocated budget. they can rent storage space processing units or even networks in which they can deploy and run their software and systems, EC2 from Amazon is an example of this type of service.

The cloud approach has attracted the attention of vehicle manufacturers and precisely the ITS field, mainly for the reason that the resources and services provided by the $\mathrm{CC}$ are highly valued by road users, for this reason, several projects have been conducted in this context to develop a new model that exploits the potential benefits of the cloud for the profit of autonomous vehicles, the best known is the Vehicular Cloud model, the subject of the next paragraph.

\subsection{Vehicular Cloud}

Olariu et al[15] introduced the idea of AVC (Autonomous Vehicular Clouds) which refers to a new concept that aims to coordinate the storage and communication resources as well as the sensing and processing capacities in order to provide them to internal and external users as a service. This new concept intends to strengthen the classic cloud infrastructure, especially since everywhere you go, there are a significant number of vehicles spending hours throughout the day in parking lots. Consequently, the resources at their disposal remain unexploited, which is a great loss. If these resources are well exploited, the $\mathrm{VC}$ can become in the nearest future a major pillar in the cloud platform.

In a VANET context, the cloud vehicle can act both as a service provider to improve the services available on the network, as well as being a service consumer by using the available data and services. In this situation, VANET is more appropriate for IaaS and SaaS, while PaaS is not applicable for it. In the case of the IaaS layer, the services provided by VANET can be Network as a Service (NaaS), since any vehicle on the road network can be considered as an Internet access point gateway, vehicles can also rent their storage or other resources on demand. For SaaS, real-time data from the network can be shared with subscribed or registered users. Besides, entertainment applications and $\mathrm{P} 2 \mathrm{P}$ services can also be delivered.

\subsection{VANET Cloud Architecture}

The VANET Cloud can be organized according to three main architectures, namely the Vehicular Cloud (VC), the Vehicular using Cloud (VuC), and finally the Hybrid Cloud (HC). Static Cloud concerns parked vehicles providing cloud services, in this case, it is the infrastructure (processing, communication, data storage) that can be rented on-demand to internal network members or to others belonging to external networks. For the Dynamic Cloud, it is constituted on demand through the ad-hoc way. $\mathrm{VuC}$ links the VANET to the traditional Cloud in which road users can take advantage of cloud services along the way such as traffic information and safety applications. And finally, for the $\mathrm{HC}$, the interaction is between the VCs and the traditional cloud for the exchange of services and information. Vehicles and RSUs will act as gateways to the traditional clouds.

\subsection{Forming VC}

The VC is constituted in the following way, first, the vehicles select the brokers among them and identify the boundaries of the cloud, then they elect among these brokers an Authorization Entity (AE) in order to apply for authorization to form a cloud. once the brokers and the $\mathrm{AE}$ have been selected. the $\mathrm{AE}$ sends invitations to the vehicle nodes to join the cloud they form on the premises without exceeding the limits of the cloud. the vehicles that are interested by this request, will reply with an ACK, in case the permitted number is exceeded, $\mathrm{AE}$ will request permission from the higher authorities to grant it the permission to create this cloud and allocate the necessary resources. After the agreement, the cloud members will share resources to form a virtual environment rich in resources and capabilities. Once the 
cloud has fulfilled its role, $\mathrm{AE}$ is charged to dissolve it[16].

\subsection{Static and Dynamic VS}

As previously mentioned, stationary and Dynamic vehicles form a group that can provide a set of services such as data storage and processing capacities, which remains the most coveted and important resource. This type of cloud platform is useful when a vehicle or user needs storage space, and processing units to store data or to run a program or application. In this way, the service consumer will delegate this task to an external entity, which, as explained in the IaaS service provider, will be responsible for its availability and security. Unlike the traditional cloud, the static/dynamic cloud allows vehicles to communicate using both V2V and V2I modes without necessarily being connected to the Internet, which therefore guarantees the availability of services such as data storage, in addition to the fact that the vehicle can be both a service provider and a service consumer.

In the automotive industry, resource management represents a major challenge, mainly because vehicles frequently generate and manipulate a large amount of data of different types that will be processed for different purposes. As mentioned above, the $\mathrm{CC}$ is a promising approach to solve this problem, except that it suffers from some limitations such as the lack of component autonomous management and flexibility of the allocated resources[17], which are considered as a requirement for the implementation of an inter-vehicle communication mechanism, hence its inadequacy with the VANET context. On the other side, the VC seems to represent an efficient solution to manage this problem, particularly since it provides mobile resources and enables the autonomous management of the available resources.

\section{Information Centric Networking (ICN)}

\subsection{Introduction}

ICN is a content-centric network architecture proposed to replace the IP Protocol on which the Internet is based. To achieve the ICN approach, several implementations have been proposed using the ICN communication paradigm, named data networking is one of them. In the case of ICN, a user who expresses an interest in content or information broadcasts its request, and any node of the network containing the desired information replies back. The objective is to dissociate the content's attachment to specific physical support and improve the network's performance. It also enables to cache of the information retrieved from the intermediate nodes during the forwarding and reception of user interests.

The named data network (NDN) is a concept adopted by the ICN, which is able to coexist with the constraints of mobility and connectivity instabilities in mobile networks such as VANET. The user can provide and consume cached content from any neighboring source node regardless of the network connectivity link between the requester and the provider.

The management of cached content has been the subject of several researches in order to provide a solution to the problem of data storage in mobile networks, and this has become clarified mainly with the emergence of the ICN. In [18] highlighted the concept of distributed caching management decision-making in order to optimize the insertion of information replicas in storage devices linked to the nodes of networks using the ICN. In another work[19], the authors implemented a mathematical framework using Markov chain in order to evaluate the caching efficiency for various caching strategies. In the same way, [20] handles the distribution of caching capacity among routers within the constraint of total storage budget in the network. A heuristic method is suggested using node centrality for the specific case of dynamic networks with frequent publishing of content. The authors conclude that the topology of the network and content popularity are two relevant factors in placing caching capacity. In another work [21], the game theory was applied to cache the popular videos at the small cell base station (SBSs). the authors attempted to optimize the average profit of the service provider by resolving a complex optimization problem using uniform and non-uniform pricing models. Similarly, [18] proposed a game-theoretical approach in the ICN to motivate owners of wireless access points to lease their unused bandwidth and data storage for the benefit of the content provider under the constraint of partial coverage.

\subsection{ICN architecture in the VANET context}

The growing interest in ICN by the scientific community has accelerated the emergence of several architectures, the Name Data Networking (NDN) is the most efficient among the ICN implementations. The NDN architecture uses a hierarchical name structure that identifies any content and delivers it back to the initiators of the request[22]. For this reason, there are two types of packets that are used, the first is an interest packet sent by the applicant when the latter needs data or information, and the second is a data packet containing the requested data constituting the response to the expressed interest.

In NDN the data packet is only sent if an interest has been received[23], such packets are considered unwanted and the intermediate nodes reject them automatically without forwarding them. Intermediate nodes act as relay points and are responsible for transferring the interest package to the content provider, then delivering the content to its requesters, and caching the content in their buffers (CS content stores). On the other side, the content name is loaded in both data and interest packets. Interest is transferred from node to node by using only the content name until it reaches the provider or Replica node. Sometimes the data packet contains the same delivered interest and provided to the requester using the same path as the interest packet.

Concerning vehicular networks, VANET has attracted the attention of industries and the scientific 
community, as a result, several protocols and standards have emerged. In addition, due to the fact that most of the communications in VANET follow a contentoriented approach, several proposals have been presented to verify the feasibility of using ICN as the principal communication architecture[24,25]. Several solutions have been proposed to this effect, by making improvements to the NDN core and by revising the initial design and recommendations to adapt them to the VANET case [26]. Multiple communication schemes for VANET can be implemented based on an NDN communication architecture using a data-interest exchange. In such a context, vehicles can act as consumers, data relays, and providers, at the same time, base stations, RSUs, and network elements can act as brokers for transferring and caching data.

In traditional IP/TCP-based networks vehicles may request the same buffer or map to download content from the internet or view a video. Therefore, all requests must be handled by the initial provider, which is identified on the network by its IP address. In such a case, similar requests with different IP source addresses can occur in significant numbers from the vehicles, causing communication congestion and degrading network performance and quality of service. Besides, the dynamic topology due to the high mobility of vehicles makes communications more sensitive and can affect scalability negatively. On the other side, in NDN(ICN)-based networks, the communication is carried out through the name of the content and a possibility to cache the exchanged data. Separating the content from its location will improve content retrieval. The content can be delivered by any surrounding vehicle or RSUs depending on the availability and the provider's load, without forgetting that the architecture supports mobility simply and efficiently.

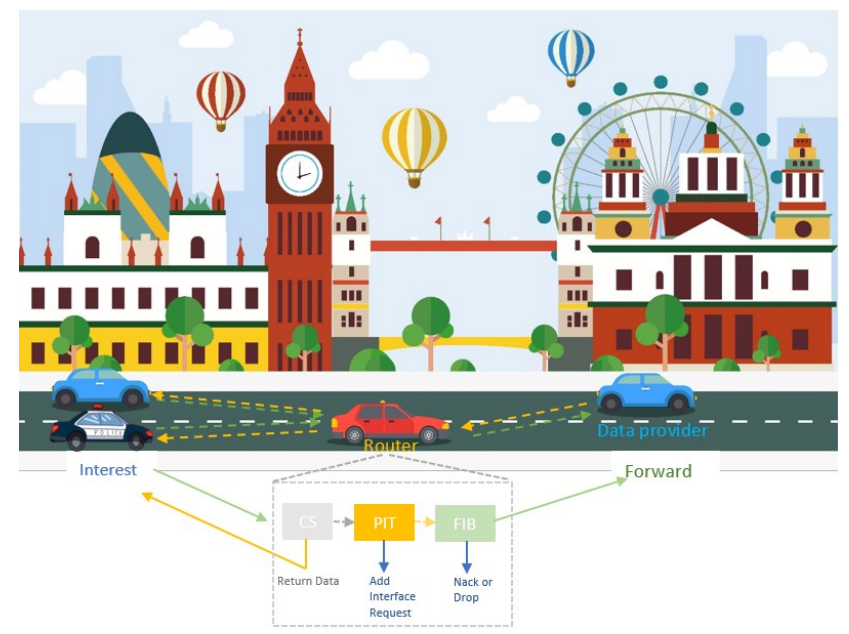

Fig. 4 : Interest routing Process

The originality of NDN(ICN) lies in the decoupling process of the content from the initial location, in addition, all security measures are applied to the content itself instead of the communication channel. Hence the interest of caching the data at the intermediate nodes, the nodes will serve to preserve the content instead of transferring it to the original provider node[27,28].
Each node of the network maintains a table called Pending Interest Table (PIT), in which interest packets are stored until the demand for that interest is satisfied by transferring the requested content to the requesting node. Each line of the PIT contains details of the incoming interest packet, namely the name of the content, the list of interfaces where the interest has been received and the timeout range for deleting it from the table. The nodes use the PIT table, to forward the content packet to the requester without going through the IP address (Fig. 5).

The second table which is also maintained by the nodes is called the Forwarding Information Base (FIB), which contains information about the next-hop node. a node uses a relatively long prefix name, in order to better select the next-hop node to reach a destination node providing the content, the routing protocol in this case is in charge of filling up the FIB table.

\section{Using UAV in the context of vehicular cloud based on ICN}

As explained before, vehicles are in constant mobility, and the available resources are not sufficient to perform several tasks at the same time (data collection, processing, filtering...), hence the importance of using the vehicle cloud (VC) approach that will allow to unify the resources and make them available to the vehicles that may request them. The ICN approach is important for the case of VANETs, especially with the emergence of $6 \mathrm{G}$, vehicles will eventually need to retrieve the data faster, the problem with intermediate nodes (routers) is that they are also mobile and the desired information can be hard to retrieve if the nodes containing the requested information are far from the research area.

That's why in our proposal we suggest using UAV as a router, especially as we explained before, it can do several tasks such as caching, processing, and packet relaying, moreover, it can perfectly collaborate with the road infrastructure units, besides, in the VANET network based on NDN/ICN, the UAV will allow improving the processing time and the storing capacity.

\subsection{Related Works}

Few studies have been conducted on improving the performance of V2X-6G systems up to now. This is due to the complexity of setting up a heterogeneous architecture that can manage a wide range of stakeholders (vehicles, devices, humans, road infrastructure...). The introduction of the IoT will further complicate the task, which will push industries to manage several use cases.

Concerning the utilization of UAVs as a relayer and as a processing element, several studies have been conducted. the study conducted [29], highlighted the advantage of using UAVs as nodes for storing, carrying and forwarding information. The purpose is to improve the continuity of path connectivity between nodes and to reduce the delay of packet delivery. In a second work[30], the authors focused on static cloud vehicles, in order to use them for data storage in a VANET network managed by NDN data exchanges. 


\subsection{Proposed Concept}

In our proposition, we aim to use the storage and processing capacities of cloud vehicles covered by UAVs, in order to lighten the load of the vehicles, which in some cases require more resources than those they currently possess. This concept also aims to externalize complex tasks that are difficult to handle by the vehicle, and also to be a substitute cloud platform that a road user can exploit to retrieve a given data or like the vehicle, to use it for performing a specific task (such as requesting more space to run a mobile application).

Our proposed smart city is equipped with all the necessary equipment in order to connect the vehicles to each other.

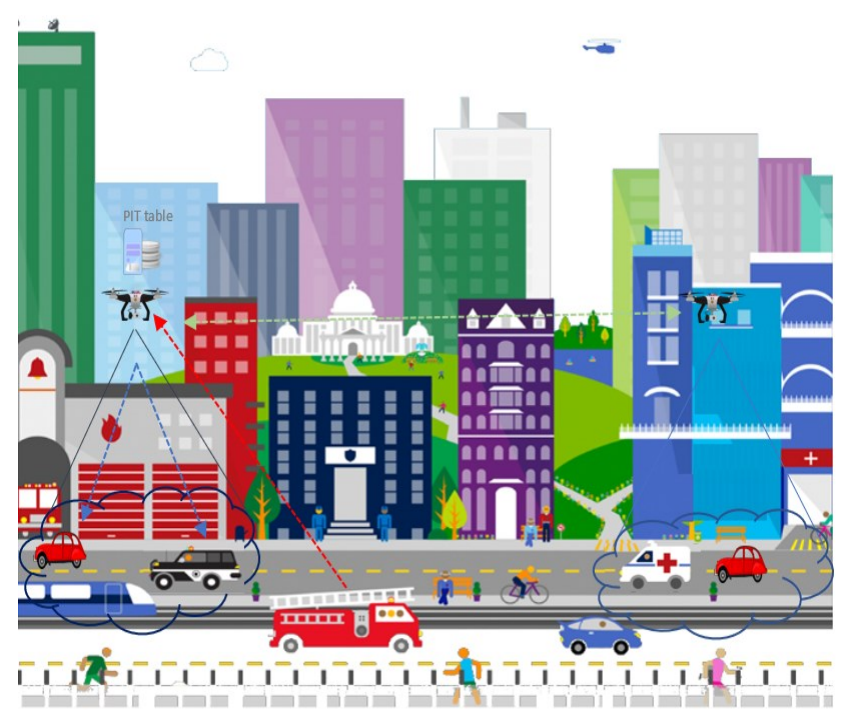

Fig. 6 : Proposed smart city

In order to access particular information, free memory space, or processing a complex task, the vehicle (or road user) submit an interest to the UAV (red arrow), which will consult the PIT table in which the interest packets are stored as well as the list of interfaces from which the interests are received, in order to identify the location of the node that meets the requirements (Fig. 7). Once the request is processed by the target node, the latter will send the response using the same path through which the interest passed. Before transferring the response to the initiator of the request, the UAV updates the PIT table with the new items. The PIT table, in addition to the information related to the interest and the interfaces node, also contains the operation type, if it is a content search, storage space, processing unit, or any other type of operation. If the UAV does not find the node that corresponds to the requirement, it will transmit the interest to the neighboring UAV (green arrow), so that the latter can do the same operation.

\subsection{Advantages of the new approach}

As we explained before, the new approach allows faster access to the target node, because the UAV will do its search in the PIT table which contains all the nodes' locations (which can belong to other VCs of another UAV). If the search is unsuccessful in the VC under the coverage of the UAV, the latter will forward the request to the neighboring UAV, and so on until it finds the appropriate target node for the requested operation.

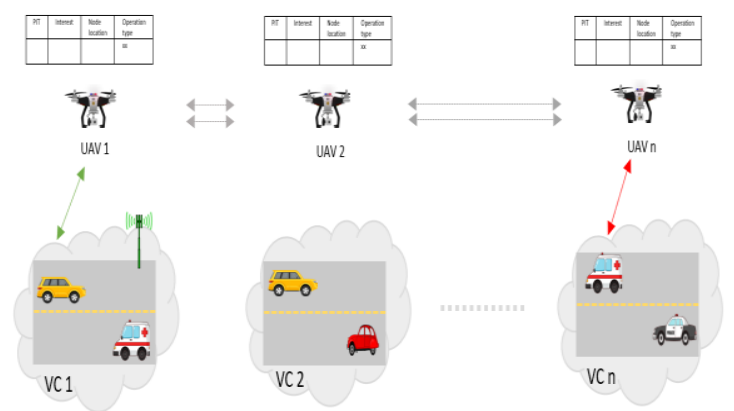

Fig. 8 : searching for the target node via the neighbouring UAV

Under these conditions, the time it takes to transmit a response from one UAV to another is given by the following formula:

$$
\sum_{k=1}^{n} a_{k} * t_{k}
$$

In the formula (1) the ' $a$ 'corresponds to the time taken by the UAV to find the node location in the PIT table, and the ' $t$ ' corresponds to the time taken to transfer the interest from one UAV to another.

Consequently, the end-to-end sending of a parquet from a node $\mathrm{x}$ to a node $\mathrm{y}$ is expressed by the following formula (2):

$$
\begin{aligned}
T_{x, y}=\sum_{k=1}^{n}\left(T_{s_{k}}\right. & \left.+T_{p_{k}}+T T_{p_{k}}+T_{Q_{k}}+a_{k} * t_{k}\right)(2) \\
& +\sum_{k=1}^{n-1} T_{c_{k}}
\end{aligned}
$$

With $\mathrm{n}$ the total number of hops, $T_{s_{k}}, T_{p_{k}}, T T_{p_{k}}, T_{Q_{k}}$ and $T_{c_{k}}$ are respectively the $\mathrm{k}$-th hop sending delay, propagation delay, processing delay, queuing delay and delivery delay.

As a result, the performed search is of linear complexity, unlike the classical ICN search which is similar to a binary tree search with variable arc weights (due to the mobility of the vehicles) which is characterized by an exponential complexity.

\subsection{Perspectives}

The implementation of such an architecture requires a set of resources to be deployed, the idea of including the UAV as a relay element will facilitate the retrieval of the target node containing the desired information or service, which will facilitate the vehicle's task and will save a considerable amount of time, unlike the classical ICN approach in which the vehicle takes more time and resources to find the target node.

It is also important to develop a reliable mechanism that allows the cooperation of the cloud vehicle groups (dynamic and static) with the UAVs, based on the ICN communication approach. the aim is to validate this model by simulating the exchanges between the different 
actors and compare the response time with a VANET and ICN-based model.

\section{Conclusion}

In this article we highlight the new V2X networks based on $6 \mathrm{G}$, we discover the different technologies that can be exploited for the benefit of V2X networks and more specifically for VANET networks in order to improve the capacity of the network and improve the quality of service offered to its customers. In the second part, we explore the VANET network characteristics, and we focus on the advantage of using the vehicular cloud evolving in an ICN context. In the last part we propose a new approach, which is a combination of the vehicular cloud with ICN and UAV one of the leading technologies of the $6 \mathrm{G}$.

The study conducted in this paper will serve us in future works, which are oriented towards the improvement of services available in vehicular networks on a smart city. The purpose is to allow vehicles that have a certain level of autonomy to drive in the city safely while experiencing the best possible services.

\section{References}

1. S. Chen, J. Hu, Y. Shi, Y. Peng, J. Fang, R. Zhao, and L. Zhao, IEEE Comm. Stand. Mag. 1, 70 (2017)

2. F. Tariq, M. R. A. Khandaker, K.-K. Wong, M. A. Imran, M. Bennis, and M. Debbah, IEEE Wireless Commun. 27, 118 (2020)

3. W. Saad, M. Bennis, and M. Chen, IEEE Network 34, 134 (2020)

4. I. F. Akyildiz, A. Kak, and S. Nie, IEEE Access 8, 133995 (2020)

5. L. Bi, X.-A. Fan, N. Luo, K. Jie, Y. Li, and Y. Liu, IEEE Trans. Intell. Transport. Syst. 14, 1996 (2013)

6. O. Semiari, W. Saad, M. Bennis, and B. Maham, IEEE Trans. Wireless Commun. 17, 779 (2018)

7. X. Cao, P. Yang, M. Alzenad, X. Xi, D. Wu, and H. Yanikomeroglu, IEEE J. Select. Areas Commun. 36, 1907 (2018)

8. H. Menouar, I. Guvenc, K. Akkaya, A. S. Uluagac, A. Kadri, and A. Tuncer, IEEE Commun. Mag. 55, 22 (2017)

9. S. Al-Sultan, M. M. Al-Doori, A. H. Al-Bayatti, and H. Zedan, Journal of Network and Computer Applications 37, 380 (2014)

10. M. S. Sheikh and J. Liang, Wireless Communications and Mobile Computing 2019, 1 (2019)

11. M. N. Mejri, J. Ben-Othman, and M. Hamdi, Vehicular Communications 1, 53 (2014)

12. X. Li, J. Liu, X. Li, and W. Sun, in 2013 5th International Conference on Intelligent Networking and Collaborative Systems (IEEE, Xi'an City, Shaanxi Province, China, 2013), pp. 210-214
13. T. Rodmunkong, P. Wannapiroon, and P. Nilsook, IJSPS 2, (2014)

14. S. Shahzadi, M. Iqbal, Z. U. Qayyum, and T. Dagiuklas, in 2017 IEEE 22nd International Workshop on Computer Aided Modeling and Design of Communication Links and Networks (CAMAD) (IEEE, Lund, Sweden, 2017), pp. 1-6

15. M. Abuelela and S. Olariu, in Proceedings of the 8th International Conference on Advances in Mobile Computing and Multimedia - MoMM '10 (ACM Press, Paris, France, 2010), p. 6

16. H. Vasudev and D. Das, in 2019 International Conference on Information Networking (ICOIN) (IEEE, Kuala Lumpur, Malaysia, 2019), pp. 72-77

17. A. Boukerche and R. E. De Grande, Computer Networks 135, 171 (2018)

18. V. Sourlas, L. Gkatzikis, P. Flegkas, and L. Tassiulas, IEEE Trans. Netw. Serv. Manage. 10, 286 (2013)

19. Xu Zhang, Ning Wang, V. G. Vassilakis, and M. P. Howarth, in 2014 International Conference and Workshop on the Network of the Future (NOF) (IEEE, Paris, France, 2014), pp. 1-5

20. Y. Wang, Z. Li, G. Tyson, S. Uhlig, and G. Xie, IEEE Trans. Comput. 65, 95 (2016)

21. J. Li, H. Chen, Y. Chen, Z. Lin, B. Vucetic, and L. Hanzo, IEEE J. Select. Areas Commun. 34, 2115 (2016)

22. B. Nour, K. Sharif, F. Li, H. Khelifi, and H. Moungla, in 2018 IEEE Conference on Standards for Communications and Networking (CSCN) (IEEE, Paris, 2018), pp. 1-6

23. B. Ahlgren, C. Dannewitz, C. Imbrenda, D. Kutscher, and B. Ohlman, IEEE Commun. Mag. 50, 26 (2012)

24. M. Amadeo, C. Campolo, and A. Molinaro, in Proceedings of the Ninth ACM International Workshop on Vehicular Inter-Networking, Systems, and Applications - VANET'12 (ACM Press, Low Wood Bay, Lake District, UK, 2012), p. 99

25. R. W. L. Coutinho, A. Boukerche, and A. A. F. Loureiro, IEEE Commun. Mag. 56, 85 (2018)

26. R. Hussain, S. H. Bouk, N. Javaid, A. M. Khan, and J. Lee, IEEE Commun. Mag. 56, 168 (2018)

27. A. Karami and M. Guerrero-Zapata, Computer Networks 80, 51 (2015)

28. Le Phong Du, Tuan-Anh Le, Nguyen Duc Thai, and Phuong Luu Vo, in 2017 International Conference on Information Networking (ICOIN) (IEEE, Da Nang, Vietnam, 2017), pp. 628-632

29. W. Fawaz, R. Atallah, C. Assi, and M. Khabbaz, IEEE Access 5, 23710 (2017)

30. A. Houari, T. Mazri, and A. Addaim, in 2020 Fourth International Conference On Intelligent Computing in Data Sciences (ICDS) (IEEE, Fez, Morocco, 2020), pp. 1-7 